\title{
Does levonorgestrel-releasing intrauterine system increase breast cancer risk in peri-menopausal women? An HMO perspective
}

\author{
Nava Siegelmann-Danieli ${ }^{1}$ (i) Itzhak Katzir $^{2} \cdot$ Janet Vesterman Landes $^{2}$ • \\ Yaakov Segal ${ }^{1} \cdot$ Rachel Bachar $^{1} \cdot$ Hadas Rotem Rabinovich $^{2} \cdot$ Martin Bialik $^{2}$. \\ Joseph Azuri' ${ }^{1,3} \cdot$ Avi Porath $^{1} \cdot$ Yossef Lomnicky $^{2}$
}

Received: 10 May 2017/ Accepted: 30 August 2017/Published online: 14 September 2017

(c) The Author(s) 2017. This article is an open access publication

\begin{abstract}
Purpose To evaluate the association between levonorgestrel-releasing intrauterine system (LNG-IUS) use and breast cancer (BC) risk.

Methods A cohort of all Maccabi Healthcare Services (MHS) female members aged 40-50 years between 1/2003 and $12 / 2013$ was used to identify LNG-IUS users as "cases," and 2 age-matched non-users as "controls." Exclusion criteria included: prior BC diagnosis, prior (5 years pre-study) and subsequent treatment with other female hormones or prophylactic tamoxifen. Invasive tumors were characterized by treatments received (chemotherapy, hormonal therapy, trastuzumab, or combination thereof).

Results The analysis included 13,354 LNG-IUS users and 27,324 controls (mean age: $44.1 \pm 2.6$ vs. $44.9 \pm 2.8$ years; $p<0.0001)$. No significant differences in 5-year KaplanMeier (KM) estimates for overall BC risk or ductal carcinoma in situ occurrence were observed between groups. There was a trend towards higher risk for invasive BC in LNG-IUS users (5-year KM-estimate: $1.06 \%$ vs. $0.93 \% ; p=0.051$ ). This difference stemmed primarily from the younger women (40-45 years; $0.88 \%$ vs. $0.69 \%, p=0.014$ ), whereas in older women (46-50 years), it was non-significant $(1.44 \%$ vs. $1.21 \% ; p=0.26)$. Characterization of invasive $\mathrm{BC}$ by
\end{abstract}

Nava Siegelmann-Danieli

danieli_na@mac.org.il

1 Department of Professional Medicine, Maccabi Healthcare Services, 27 Hamered St., 68125 Tel Aviv, Israel

2 Department of Pharmacy, Maccabi Healthcare Services, 27 Hamered St., 68125 Tel Aviv, Israel

3 Sackler Faculty of Medicine, Tel Aviv University, Ramat Aviv, POB 39040, 6997801 Tel Aviv, Israel treatment demonstrated that LNG-IUS users had similar proportions of tumors treated with hormonal therapy, less tumors treated with trastuzumab, $(7.5 \%$ vs. $14.5 \%)$ and more tumors treated with chemotherapy alone $(25.8 \%$ vs. $14.9 \%$; $p=0.041$ ).

Conclusions In peri-menopausal women, LNG-IUS was not associated with an increased total risk of $\mathrm{BC}$, although in the subgroup of women in their early 40's, it was associated with a slightly increased risk for invasive tumors.

Keywords Breast cancer - Contraceptive - Levonorgestrelreleasing intrauterine system $\cdot$ Peri-menopause

\section{Introduction}

"Why me and where did I go wrong?" is a question that haunts most women when hit with breast cancer (BC) diagnosis. Although there is no specific answer in the majority of cases, female gender and increasing age are established key risk factors. Genetic background and factors related to lifetime exposure to female reproductive hormones also play a role (reviewed by Howell et al. [1]). In post-menopausal women, the potential risk associated with exogenous reproductive hormones was investigated in two large prospective randomized trials initiated by the women's health initiative (WHI). While estrogen alone did not increase BC risk in women with hysterectomy, it was the combination of estrogen with progestin used in women with intact uterus which increased BC risk [2-6]. In perimenopausal women, the endogenous progestin level declines steadily [7], raising the question of whether exogenous progestin may increase $\mathrm{BC}$ risk. 
Levonorgestrel-releasing intrauterine system (LNGIUS; Mirena ${ }^{\circledR}$, Bayer HealthCare Pharmaceuticals Inc., Whippany, NJ) is an intrauterine system that releases LNG (a progestin) at an initial release rate of $20 \mu \mathrm{g} / \mathrm{day}$. It is approved by the Food and Drug Administration (FDA) as an intrauterine contraceptive for up to 5 years and for treatment of menorrhagia (heavy menstrual bleeding) [8]. Data on the incidence of cancer and particularly $\mathrm{BC}$ in women of fertile age using LNG-IUS are inconsistent. Two large retrospective studies (involving $>17,000$ LNG-IUS users, and $>25,000 \mathrm{BC}$ cases/controls) demonstrated no increase in BC risk with LNG-IUS, whereas two database studies from Finland involving $>93,000$ LNG-IUS users demonstrated an increased BC risk [9-12]. To date, the published studies were all in Finnish/German population, included a variety of ages, and did not control for prior or concurrent and subsequent hormonal interventions.

LNG-IUS has been covered in Israel under the National Health Insurance Law since March 2001 for women $\geq 45$ years with menorrhagia, although, in practice, it has been used commonly in pre- and peri-menopausal women for contraception in which case it is not reimbursed under the Health Insurance Law. Maccabi Healthcare Services (MHS), the second largest health maintenance organization (HMO) in Israel insuring approximately 2.0 million individuals, extended coverage of LNG-IUS to younger patients with various indications and it is therefore assumed that almost all MHS members who purchase LNG-IUS do so through the HMO.

The current work was designed to evaluate the incidence of BC in peri-menopausal women (MHS members) using LNG-IUS versus age-matched controls, focusing on women who were not exposed to other hormone therapies in the 5 years preceding inclusion and during study period till last follow-up. We also aimed to compare tumor characteristics and treatments received in $\mathrm{BC}$ diagnosed in LNG-IUS users versus controls.

\section{Materials and methods}

\section{Study design and patients}

In this retrospective cohort study, LNG-IUS users (cases) and age-matched controls were selected from 338,184 women who were 40-50 year old between January 2003 and December 2013. Cases included all MHS members who purchased LNG-IUS in this timeframe. The controls (2 per case) were selected sequentially (starting January 2003 forward) to be aged-matched \pm 2 years (based on calendar year) with the same exclusion criteria. Follow-up of participants was initiated (Day 1) at time of LNG-IUS insertion (cases) or the date they reached the age of the matching case (controls). Exclusion criteria included: BC diagnosis at any time before study initiation or within 6 months of inclusion, exposure to female hormones in the form of oral contraceptives (OC), fertility drugs, or hormone-replacement therapy (HRT), or prophylactic use of tamoxifen in the 5 years preceding Day 1 and through November 2015. We used Kaplan-Meier (KM) curves to estimate the 5-year BC risk in order to correct for differences in follow-up time between cases and controls (as controls were identified from 2003 forwards resulting in longer follow-up).

Data for all participants were collected from the MHS data system which captures patients' information electronically and vital status as provided by the Israeli National Insurance Institute. Systemic treatments for study participants, who were diagnosed with invasive BC during the study period, were extracted from the MHS system which captures pharmacy purchases and hospital claims.

The study was approved by the institutional review board of MHS; informed consent was waived due to the retrospective nature of the study.

\section{Breast cancer categorization}

Categorization to invasive vs. ductal carcinoma in situ (DCIS) was based on biopsy results as captured in the MHS cancer registry. Invasive BC tumors were characterized by treatments received (chemotherapy, hormonal therapy, trastuzumab, or combination thereof).

\section{Statistical analysis}

Descriptive statistics were used to summarize patient and tumor characteristics and treatments received. The Chisquared and Wilcoxon rank-sum tests were used to compare cases and controls with respect to patient and tumor characteristics. KM plots and log-rank tests were used to compare risk of BC. Five-year KM estimates for BC risk and standard errors (SE) were calculated. All statistical analyses were performed with JMP 12.0 (SAS Institute Inc., Cary, NC). Two-sided $p<0.05$ was considered statistically significant.

\section{Results}

\section{Study population}

Between January 2003 and December 2013, a total of 338,184 women in the MHS database were between the ages of 40 and 50 years. During this time period, 17,667 of these women purchased LNG-IUS through MHS, of whom 13,354 met study criteria. The age-matched control group 
included 27,324 women. The mean (SD) age was 44.1 (2.6) years in the LNG-IUS users group and 44.9 (2.8) years in the controls $(p<0.0001$; Wilcoxon rank-sum test). The LNG-IUS users group had higher proportion of younger patients compared to the controls (LNG-IUS users: $67.4 \%$ were 40-45 years of age and $32.6 \%$ were $46-50$ years of age; in the controls, the proportions were 54.4 and $45.6 \%$, respectively; $p<0.0001$, Chi-square test). As matched-age controls were identified sequentially from the beginning of the study period, follow-up was significantly longer in the control group [median (range) of $9.6(0.5-12.9)$ years vs. $6.5(0.5-12.9)$ years; $p<0.0001$, Wilcoxon rank-sum test].

\section{Breast cancer risk}

Overall, there were 223 documented BC cases in LNG-IUS users and 565 in the controls. Median (range) time from study entry to BC diagnosis was $4.3(0.5-12.3)$ years in LNG-IUS users and $5.0(0.5-12.7)$ years in the controls $(p=0.0038$; Wilcoxon rank-sum test). Within 5 years of study entry, there were $136(1.0 \%)$ documented BC cases in LNG-IUS users and 283 (1.0\%) documented BC cases in the controls. In the LNG-IUS users, these included 16 DCIS and 120 invasive tumors; in the controls, these included 42 DCIS and 241 invasive tumors.

$\mathrm{KM}$ curves for overall BC risk for LNG-IUS users and controls were not statistically significantly different (Fig. 1a), as were KM curves for DCIS risk and invasive $\mathrm{BC}$ risk (Fig. 1b, c). Five-year KM estimates for overall $\mathrm{BC}$ risk in LNG-IUS users and controls were $1.2 \%$ (SE $0.1 \%$ ) and $1.1 \%$ (SE $0.06 \%$ ), respectively $(p=0.23$ ). For DCIS risk, the respective values were $0.14 \%$ (SE $0.03 \%$ ) and $0.16 \%(\operatorname{SE} 0.03 \%)(p=0.22)$. There was a trend towards higher risk for invasive BC in LNG-IUS users vs. controls $(p=0.051)$, with 5-year KM estimates of $1.06 \%$ (SE $0.1 \%$ ) and $0.93 \%$ (SE $0.06 \%$ ), respectively (Table 1 ). In a subgroup analysis by age, the difference in the risk for invasive tumors seems to stem primarily from the younger age group: in patients aged $40-45$ years, a statistical significant difference was observed between LNG-IUS users and controls with 5-year KM estimates for invasive BC risk of $0.88 \%$ (SE $0.11 \%$ ) versus $0.69 \%$ (SE $0.07 \%$ ), respectively $(p=0.014)$; whereas in patients aged $46-50$ years, the 5-year KM estimates were $1.44 \%$ (SE $0.19 \%$ ) versus $1.21 \%$ (SE $0.10 \%$ ), respectively $(p=0.26)$ (Table 1 ).

\section{Invasive breast cancer: characterizing tumors by treatments received}

For the invasive $\mathrm{BC}$ cases identified within 5 years of study entry, the distribution of treatments received (chemotherapy alone, hormonal therapy alone, chemotherapy plus hormonal therapy, chemotherapy plus trastuzumab, and
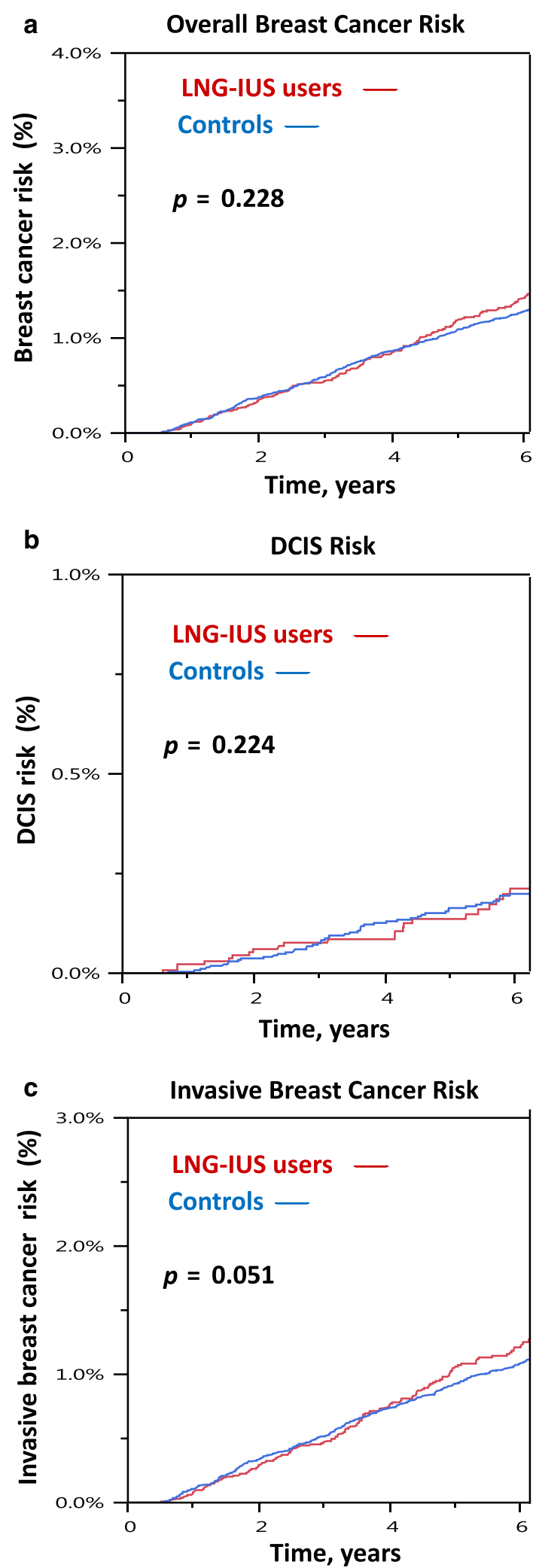

Fig. 1 KM curves for risk of developing breast cancer (a), DCIS (b), and invasive breast cancer (c) for LNG-IUS users (cases) and controls 
Table 1 Five-year KaplanMeier estimates for breast cancer risk

\begin{tabular}{llll}
\hline & LNG-IUS users & Controls & $p$ value log-rank test $^{\mathrm{a}}$ \\
\hline Breast Cancer (overall) & $1.2 \%($ SE 0.10\%) & $1.1 \%($ SE 0.06\%) & 0.23 \\
DCIS & $0.14 \%$ (SE 0.03\%) & $0.16 \%($ SE 0.03\%) & 0.224 \\
Invasive tumors & & & \\
Overall & $1.06 \%$ (SE 0.10\%) & $0.93 \%($ SE 0.06\%) & 0.051 \\
$40-45$ years & $0.88 \%$ (SE 0.11\%) & $0.69 \%($ SE 0.07\%) & 0.014 \\
$46-50$ years & $1.44 \%($ SE 0.19\%) & $1.21 \%($ SE 0.10\%) & 0.26 \\
\hline
\end{tabular}

DCIS ductal carcinoma in situ, SE standard error

${ }^{a} p$ values refer to comparing the entire KM curves (cases vs. controls) chemotherapy plus hormonal therapy plus trastuzumab) was not statistically significantly different between the LNG-IUS users and controls when all six aforementioned therapeutic options were included in the analysis ( $p=0.076$, Chi-square test; Table 2). However, when all tumors treated with trastuzumab were considered as one category (i.e., chemotherapy plus trastuzumab and chemotherapy plus hormonal therapy plus trastuzumab), a statistically significant difference in the overall distribution was observed ( $p=0.041$, Chi-square test; Table 2$)$. In addition, BC cases in LNG-IUS users were less likely to be treated with trastuzumab ( $7.5 \%$ vs. $14.5 \%)$ and more likely to be treated with chemotherapy alone $(25.8 \%$ vs. $14.9 \%)$ with the latter serving as surrogate to suggest triple-negative tumors.

\section{Discussion}

In this case-control cohort study, we showed that in perimenopausal women, LNG-IUS does not increase the risk of $\mathrm{BC}$ overall or the risk of DCIS. We did find that LNG-IUS use was associated with a slightly increased risk of invasive $\mathrm{BC}$ tumors in the subgroup of younger women (40-45 years), while no significant effect was observed in those aged $46-50$ years.

Of the four studies investigating the association between LNG-IUS use and BC, our study design is more similar to the studies reported by Backman et al. and Soini et al. $[9,11,12]$. In these studies involving 17,360 LNG-IUS users (all indications) and 93,843 LNG-IUS users (for treating/preventing menorrhagia), respectively, BC incidence was compared between LNG-IUS users and the general female population. The Backman study included LNG-IUS users of all ages with the dominant group being 35-39 years old. There was no significant difference in BC incidence between LNG-IUS users and the general female population in any of the evaluated age groups; however, in the 35-39 age group (i.e., women reaching late 30's/approximating early 40 's), the incidence was higher in LNGIUS users and the differences trended towards significance $(p=0.056)$ [9]. In the Soini study (women aged 30-49 years with an average follow-up of $>10$ years), LNG-IUS users had an increased risk for ductal breast

Table 2 Characteristics of invasive breast cancers occurring within 5 years of study entry (subtyping is approximated based on actual treatments received)

\begin{tabular}{lcc}
\hline & LNG-IUS users $(n=120)$ & Controls $(n=241)$ \\
\hline Treatment received, $n(\%)^{\mathrm{a}}$ & & $17(7.1)$ \\
Hormonal therapy alone & $12(10.0)$ & $140(58.1)$ \\
Hormonal therapy plus chemotherapy & $63(52.5)$ & $25(10.4)$ \\
Hormonal therapy plus chemotherapy plus trastuzumab & $6(5.0)$ & $10(4.1)$ \\
Chemotherapy plus trastuzumab & $3(2.5)$ & $36(14.9)$ \\
Chemotherapy alone & $31(25.8)$ & $13(5.4)$ \\
Unknown (no treatment) & $5(4.2)$ & \\
\hline
\end{tabular}

$p=0.076$, overall Chi-square test comparing the distribution of all categories; $p=0.041$, overall Chi-square test comparing distribution of all categories after combining the trastuzumab-containing categories

a Trastuzumab reimbursement was approved by Maccabi Healthcare Services in December 2015. Of the 120 invasive breast cancer patients who were LNG-IUS users 112 (93\%) were diagnosed from December 2015 forward; of the 241 controls with invasive breast cancer, 194 (80\%) were diagnosed from December 2015 forward 
cancer (standardized incidence ratio [SIR] 1.20) and lobular breast cancer (SIR 1.33; latter mostly luminal subtypes) [11]. As BC below the age of 35 is rare [13], we assume that the observed increased risk stems from patients $\geq 35$ years who constituted $80 \%$ of the study population [11]. Another study by Dinger et al. involving 5113 BC cases and 20,452 controls below the age of 50 years, found no increased BC risk with LNG-IUS users vs. copper intrauterine device (IUD) users [10]. The Dinger et al. study is different in its design from the other studies, as it was a case-control study comparing risk factor between identified BC patients and controls (matched by age and area of residence) who responded to questionnaires [10].

Our study strengths as compared with the aforementioned studies, include the careful comparison between LNG-IUS users to actual controls (not general population), excluding individuals with prior or concurrent diagnosed $\mathrm{BC}$, exclusion of women with prior/current/subsequent exposure to exogenous reproductive hormones, and using $\mathrm{KM}$ estimates to address potential biases related to differences in follow-up times. The follow-up time in our study (median of 6.5 years in LNG-IUS users and 9.6 years in the controls) is adequate for detecting increased BC risk, as studies involving other exogenous hormones in perimenopausal women (OC) and post-menopausal women (HRT) have shown that the increased breast cancer risk occurs within the first few years of exposure. With OC, the increased $\mathrm{BC}$ risk is with current/recent (within a year) of OC use [14, 15]. In HRT users, the increased BC risk occurs during the intervention phase (within 3 years) and the effect remains apparent in the post-intervention phase through 5.5 years [3]. Nonetheless, long follow-up to determine long-term effect is warranted. In addition, our study analyzed a relatively large cohort where for each individual patient treatments and clinical outcomes are known. Furthermore, our cohort represents real-life clinical practice on a national level that is not specific to menorrhagia, and thus more representative of LNG-IUS global use. Our study is also the first to characterize the invasive tumors identified in these women by treatment received. It is important to note that $\mathrm{BC}$ is not one disease but is considered to include several subtypes [16, 17], and unlike numerous studies demonstrating an association between $\mathrm{BC}$ and exposure to female hormones [18], no such association between hormonal-driven disease (i.e., luminal tumors treated with hormonal therapy) and LNG-IUS could be documented here. We are also the first to investigate the association between LNG-IUS use and BC in another geographic region (most studies thus far were performed in Finland).

The limitations of our work include its retrospective non-randomized design. Thus, selection bias may exist, since clinicians may have elected different contraceptive approaches to women with family history of BC. Also, the information regarding the indication for the LNG-IUS use in each case was not available (though it was estimated by two Gynecology directors [YS and RB personal information] that $90 \%$ of LNG-IUS users in Israel use it for contraception). The MHS data system does not include information on patients who may have purchased LNGIUS not through MHS/MHS-affiliated pharmacies; however, we assume that as it is covered by MHS, most LNGIUS users did purchase it through MHS. Only a few women had more than one LNG-IUS inserted. The recommended LNG-IUS use is 5 years and the median follow-up in LNGIUS users was 6.5 years. Also, in our analyses, we used age as a proxy for menopausal state. It is likely that in the LNG-IUS users, the majority of women were premenopausal, as women received it for contraception or menorrhagia. For the controls, the menopausal state is unknown, although we do know that the mean age of menopause in Israel is 51. In addition, pathological information was not available so tumor subtyping could not be performed. It should be noted, though, that in Israel, hormonal therapy and trastuzumab treatment do require pathological information for approval. Lastly, MHS approved trastuzumab reimbursement for human epidermal growth factor receptor 2 (Her2)-positive invasive BC in December 2005, whereas our study included women who were eligible (age wise) between January 2003 and December 2013. Notably, the majority of the invasive BC patients in our study were diagnosed after trastuzumab reimbursement approval (LNG-IUS users, 93\%; controls, $80 \%)$.

In conclusion, our study of a large cohort of LNG-IUS users and age-matched controls demonstrates that in perimenopausal women, LNG-IUS use is not associated with an increased risk of $\mathrm{BC}$ overall; although in younger women (40-45 years) LNG-IUS use may be associated with a slightly increased risk for invasive BC. More tumors treated with chemotherapy alone, and fewer tumors treated with trastuzumab were identified among LNG-IUS users, and there were similar proportions of patients treated with hormonal therapy. Further study on the impact of LNGIUS use on BC risk by age, and analysis of $\mathrm{BC}$ subtypes is warranted.

Acknowledgements Third-party medical writing assistance and statistical analysis assistance were provided by Avital Bareket-Samish, $\mathrm{PhD}$, of BioInsight Ltd. and were funded by Bayer Israel Ltd.

\section{Compliance with ethical standards}

Conflict of interest The authors declare no conflict of interest.

Open Access This article is distributed under the terms of the Creative Commons Attribution 4.0 International License (http://crea tivecommons.org/licenses/by/4.0/), which permits unrestricted use, 
distribution, and reproduction in any medium, provided you give appropriate credit to the original author(s) and the source, provide a link to the Creative Commons license, and indicate if changes were made.

\section{References}

1. Howell A, Anderson AS, Clarke RB, Duffy SW, Evans DG, Garcia-Closas M, Gescher AJ, Key TJ, Saxton JM, Harvie MN (2014) Risk determination and prevention of breast cancer. Breast Cancer Res 16(5):446. doi:10.1186/s13058-014-0446-2

2. Chlebowski RT, Anderson GL (2012) Changing concepts: menopausal hormone therapy and breast cancer. J Natl Cancer Inst 104(7):517-527. doi:10.1093/jnci/djs014

3. Chlebowski RT, Rohan TE, Manson JE, Aragaki AK, Kaunitz A, Stefanick ML, Simon MS, Johnson KC, Wactawski-Wende J, O'Sullivan MJ, Adams-Campbell LL, Nassir R, Lessin LS, Prentice RL (2015) Breast cancer after use of estrogen plus progestin and estrogen alone: analyses of data from 2 women's health initiative randomized clinical trials. JAMA Oncol 1(3):296-305. doi:10.1001/jamaoncol.2015.0494

4. Anderson GL, Limacher M, Assaf AR, Bassford T, Beresford SA, Black H, Bonds D, Brunner R, Brzyski R, Caan B, Chlebowski R, Curb D, Gass M, Hays J, Heiss G, Hendrix S, Howard BV, Hsia J, Hubbell A, Jackson R, Johnson KC, Judd H, Kotchen JM, Kuller L, LaCroix AZ, Lane D, Langer RD, Lasser N, Lewis CE, Manson J, Margolis K, Ockene J, O'Sullivan MJ, Phillips L, Prentice RL, Ritenbaugh C, Robbins J, Rossouw JE, Sarto G, Stefanick ML, Van Horn L, Wactawski-Wende J, Wallace R, Wassertheil-Smoller S, Women's Health Initiative Steering Committee (2004) Effects of conjugated equine estrogen in postmenopausal women with hysterectomy: the women's health initiative randomized controlled trial. JAMA 291(14):1701-1712. doi:10.1001/jama.291.14.1701

5. Anderson GL, Chlebowski RT, Aragaki AK, Kuller LH, Manson JE, Gass M, Bluhm E, Connelly S, Hubbell FA, Lane D, Martin L, Ockene J, Rohan T, Schenken R, Wactawski-Wende J (2012) Conjugated equine oestrogen and breast cancer incidence and mortality in postmenopausal women with hysterectomy: extended follow-up of the women's health initiative randomised placebocontrolled trial. Lancet Oncol 13(5):476-486. doi:10.1016/ S1470-2045(12)70075-X

6. Chlebowski RT, Anderson GL, Gass M, Lane DS, Aragaki AK, Kuller LH, Manson JE, Stefanick ML, Ockene J, Sarto GE, Johnson KC, Wactawski-Wende J, Ravdin PM, Schenken R, Hendrix SL, Rajkovic A, Rohan TE, Yasmeen S, Prentice RL, Investigators WHI (2010) Estrogen plus progestin and breast cancer incidence and mortality in postmenopausal women. JAMA 304(15):1684-1692. doi:10.1001/jama.2010.1500
7. O'Connor KA, Ferrell R, Brindle E, Trumble B, Shofer J, Holman DJ, Weinstein M (2009) Progesterone and ovulation across stages of the transition to menopause. Menopause 16(6):1178-1187. doi:10.1097/gme.0b013e3181aa192d

8. Mirena (2015) Package insert. Bayer HealthCare Pharmaceuticals Inc., Whippany, NJ

9. Backman T, Rauramo I, Jaakkola K, Inki P, Vaahtera K, Launonen A, Koskenvuo M (2005) Use of the levonorgestrel-releasing intrauterine system and breast cancer. Obstet Gynecol 106(4):813-817. doi:10.1097/01.AOG.0000178754.88912.b9

10. Dinger J, Bardenheuer K, Minh TD (2011) Levonorgestrel-releasing and copper intrauterine devices and the risk of breast cancer. Contraception 83(3):211-217. doi:10.1016/j.contra ception.2010.11.009

11. Soini T, Hurskainen R, Grenman S, Maenpaa J, Paavonen J, Joensuu H, Pukkala E (2016) Levonorgestrel-releasing intrauterine system and the risk of breast cancer: a nationwide cohort study. Acta Oncol 55(2):188-192. doi:10.3109/0284186X. 2015.1062538

12. Soini T, Hurskainen R, Grenman S, Maenpaa J, Paavonen J, Pukkala E (2014) Cancer risk in women using the levonorgestrelreleasing intrauterine system in Finland. Obstet Gynecol 124(2 Pt 1):292-299. doi:10.1097/AOG.0000000000000356

13. DeSantis C, Ma J, Bryan L, Jemal A (2014) Breast cancer statistics, 2013. CA Cancer J Clin 64(1):52-62. doi:10.3322/caac. 21203

14. Nelson HD, Zakher B, Cantor A, Fu R, Griffin J, O’Meara ES, Buist DS, Kerlikowske K, van Ravesteyn NT, Trentham-Dietz A, Mandelblatt JS, Miglioretti DL (2012) Risk factors for breast cancer for women aged 40 to 49 years: a systematic review and meta-analysis. Ann Intern Med 156(9):635-648. doi:10.7326/ 0003-4819-156-9-201205010-00006

15. Beaber EF, Buist DS, Barlow WE, Malone KE, Reed SD, Li CI (2014) Recent oral contraceptive use by formulation and breast cancer risk among women 20 to 49 years of age. Cancer Res 74(15):4078-4089. doi:10.1158/0008-5472.CAN-13-3400

16. Perou CM, Jeffrey SS, van de Rijn M, Rees CA, Eisen MB, Ross DT, Pergamenschikov A, Williams CF, Zhu SX, Lee JC, Lashkari D, Shalon D, Brown PO, Botstein D (1999) Distinctive gene expression patterns in human mammary epithelial cells and breast cancers. Proc Natl Acad Sci USA 96(16):9212-9217

17. van't Veer LJ, Dai H, van de Vijver MJ, He YD, Hart AA, Mao M, Peterse HL, van der Kooy K, Marton MJ, Witteveen AT, Schreiber GJ, Kerkhoven RM, Roberts C, Linsley PS, Bernards R, Friend SH (2002) Gene expression profiling predicts clinical outcome of breast cancer. Nature 415(6871):530-536. doi:10. 1038/415530a415530a

18. Anderson KN, Schwab RB, Martinez ME (2014) Reproductive risk factors and breast cancer subtypes: a review of the literature. Breast Cancer Res Treat 144(1):1-10. doi:10.1007/s10549-014$2852-7$ 